\title{
Nonlinear Dynamics Feature of Active Homing Echo Signal
}

\author{
Shen Shen ${ }^{1,3}$, Cheng Hong ${ }^{2}$,Wang Mingzhou ${ }^{1,3}$ \\ ${ }^{1}$ The 705 Research Institute, China Shipbuilding Industry Corporation, Xi'an 710075, China. \\ ${ }^{2}$ The 705 Proving Ground, China Shipbuilding Industry Corporation, Kunming 650051, China. \\ ${ }^{3}$ Science and Technology on Underwater Information and Control Laboratory, Xi'an 710075, China.
}

\begin{abstract}
Based on the nonlinear dynamics theory, the feature of the sinusoidal signal, the active homing echo signal and the active homing echo signal after filtering and normalizing is presented detailedly in this paper. Through the analysis of the phase trajectory, the phase trajectory slope and the slope spectrum's changing rule of a certain number of measured active homing echo signal sample data, it can be seen that the phase trajectory motion characteristic, the slope and the slope spectrum's changing rule have certain separability.

Index Terms - active homing echo signal, nonlinear, phase trajectory, slope
\end{abstract}

\section{Introduction}

In torpedo homing signal detection, the echo signal which is received by homing system includes the target echo signal, the radiated noise, the torpedo self-noise, the environmental noise, etc. Weak target echo signal usually covered by some kinds of interference noise, which makes the target echo signal detection difficult. Traditionally, the correlation function method in time domain and the power spectrum method in frequency domain are used to signal characteristic extraction. In recent years, with the development of the nonlinear dynamics theory, some people found that the signals which are generally considered to be random and non-stationary have the nonlinear characteristics, active homing echo signal is one of them. In this paper, based on the nonlinear dynamics theory, some nonlinear features of sinusoidal signal and the active homing echo signal after filter processing are researched, also the further discussion of the change rule of its phase trajectory slope is given. The simulation results show that the homing echo signal has the nonlinear characteristics, its phase trajectory movement rule is similar to that of the sinusoidal signal, and its nonlinear slope characteristic has certain separability.

\section{The Phase Trajectory Characteristic of the Sinusoidal Signal}

The sinusoidal signal is one kind of single with the most single frequency components. Through the Fourier transform, any complex signals can be decomposed into the superposition of sinusoidal signal with different frequencies and amplitudes. Sinusoid signal can be expressed as:

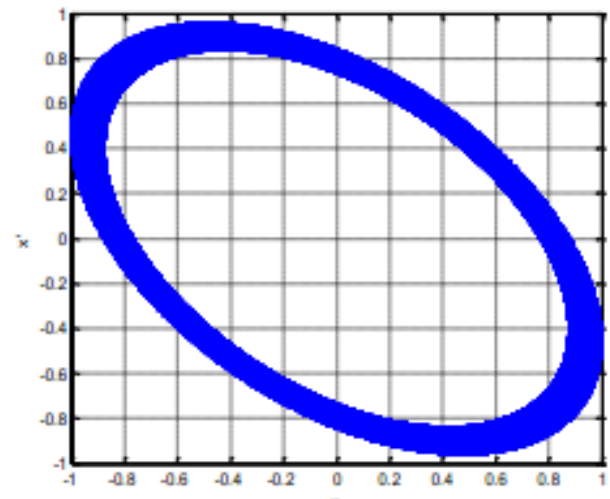

Figure 1 the phase track of sinusoidal signal

$$
x(t)=A \sin \left(2 \pi f_{0} t+\varphi\right)
$$

Where, $A$ is the amplitude, $f_{0}$ is the signal frequency, $\varphi$ is the initial angle.

This paper mainly studies the nonlinear characteristic of the sinusoidal signal, let

$$
x^{\prime}(t)=x(t+1)-x(t)
$$

Studying the phase plane trajectory movement characteristic of $\left(x, x^{\prime}\right)$. Fixing the value of $\varphi$ and changing the value of $f_{0}$ between $18 \mathrm{kHz}$ and $22 \mathrm{kHz}$. Through simulation it can be seen that the phase trajectories of the sinusoidal signal are all similar, the phase track of $\left(x, x^{\prime}\right)$ is similar to oval. Figure 1 shows the sinusoidal phase trajectory when $\varphi$ is equal to $45^{\circ}$.

\section{The Phase Trajectory Characteristic of the Active Homing Echo Signal}

The original sample data is the experimental measured active homing echo signal, which includes the reverberation, the environment noise, the target echo signal, etc. Known that the signal transmitted by homing system is rectangular wave, the pulse width is $24 \mathrm{~ms}$, the sampling frequency is $20 \mathrm{kHz}$, data is complex form 10 periods of active homing echo signal data were collected in all.

Four periods of the measured sample data are selected to process. Firstly the original measured data are filtered to reserve the target echo signal only. Then the sliding window 
FFT transform processing is performed for each periods signal to determine whether they contain the target echo signal or not. According to the pulse width of the homing signal, the sliding window length of the FFT transform is set to 480 , and the window is slided a quarter each time, so the whole periods data can be divided into several groups. Three groups with echo signal and three groups without echo signal are selected randomly from each periods data respectively to observe the phase track movement rule. Figure 2 shows the six groups' phase tracks of the first periods of the measured signal after filtering process.
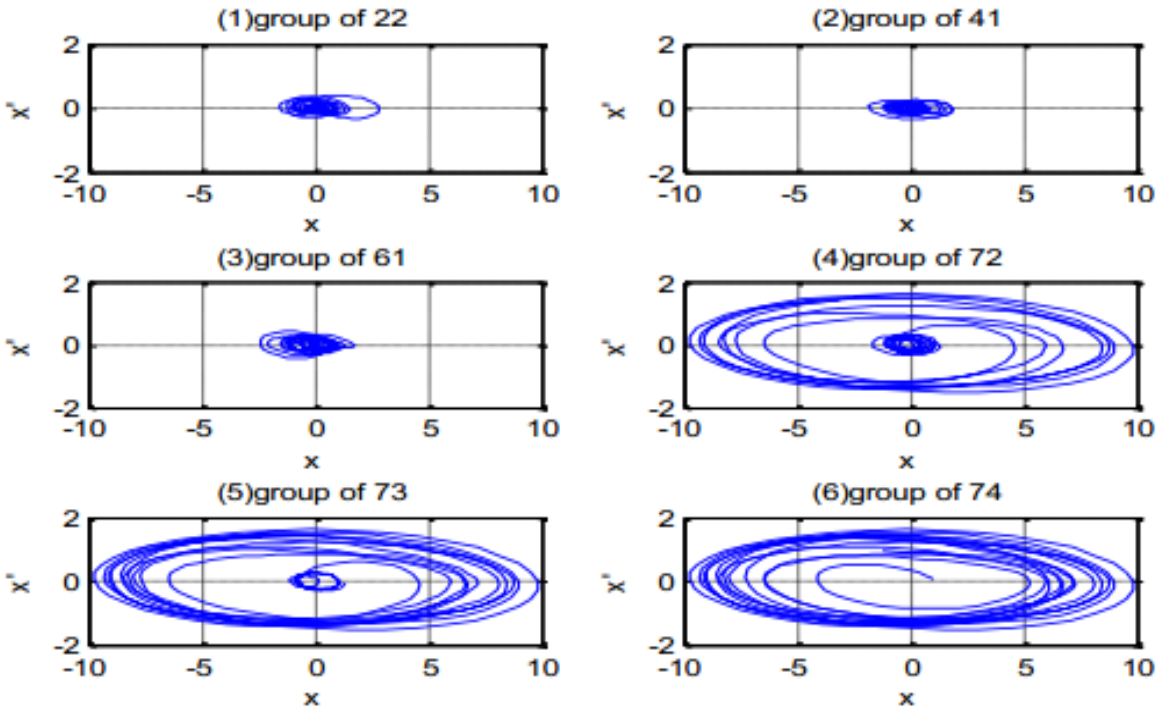

Figure 2 the phase track of the first period signal after filtering treatment

From figure 2 it can be seen that the phase tracks of the groups with target echo signal (group of $72,73,74$ ) and the groups without the target echo signal (group of 22,41,61) are different, so it can be separated. The phase trajectories of the groups with the target echo signal are similar to the sinusoidal signal's phase trajectory, like oval orbit. The phase trajectories of the groups without the target echo signal move randomly around near the origin of coordinates, irregular. Therefore the movement characteristic of its phase track has an obvious separability.

\section{The Slope Characteristic of the Active Homing Echo Signal}

By the basic mathematical formulas, for all the complex $x$, have

$$
\sin ^{\prime}(x)=\cos (x)
$$

And the amplitude of $\sin (x)$ and $\cos (x)$ are between $(-1,1)$. Therefore, in order to identify better the target echo signal, the active homing echo signal after filtering processing of each cycle is normalized to $(-1,1)$. Then according to the above steps to process the new data, and observe the rule of the phase trajectory change and the slope change. Figure 3 shows the six groups' phase tracks of the original data after filtering and normalizing process of the first periods. Figure 4 shows the six groups' phase track slope change of the original data after filtering and normalizing process of the first periods.

It can be seen from figure 3 and figure 4, that the phase trajectory of the groups with the target echo signal assumes the circular motion, and its phase trajectory slope assumes dense, continuous and regular short curve. The phase trajectory of the groups without the target echo signal moves randomly in the phase plane, and its phase trajectory slope is random distribution, irregular.
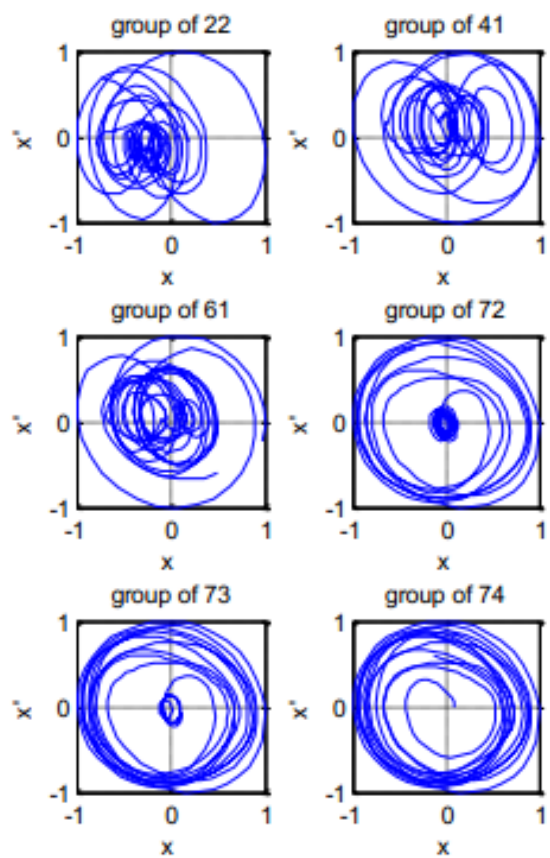

Figure 3 the phase track of the first period signal after filtering and normalizing 

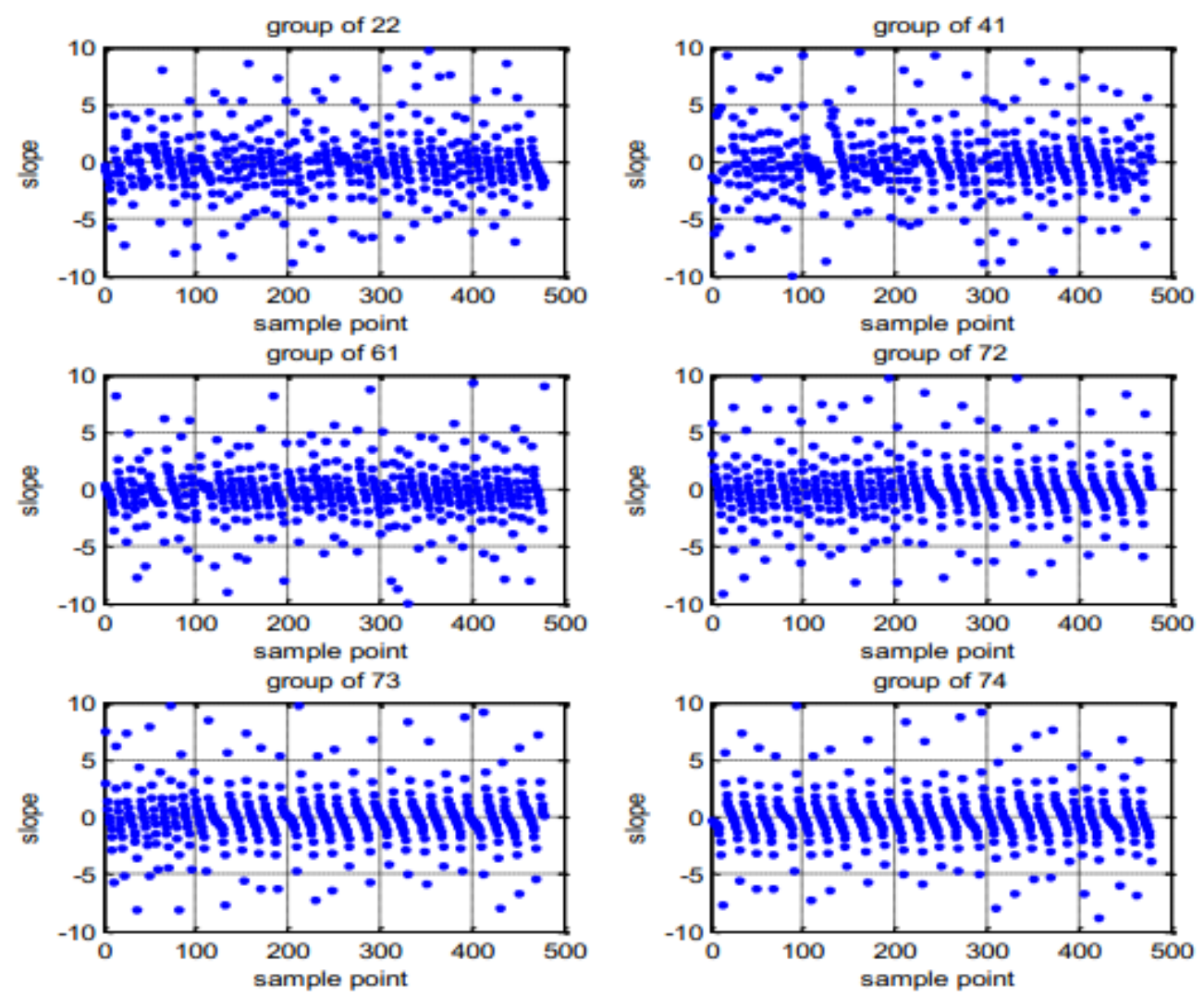

Figure 4 the phase track slope change of the original data after filtering and normalizing

FFT transform are performed to the phase trajectory slope of the active homing echo signal after filtering and normalizing treatment, to observe its spectrum. The figure 5 shows that the spectrum of the groups with the target echo signal changes are similar to the chaotic signal waveform, and the spectrum of the groups without the target echo signal changes are similar to the white noise waveform.
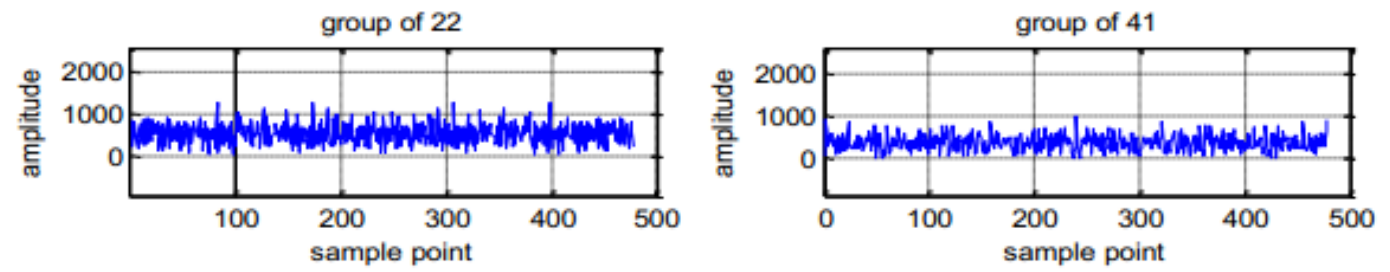

group of 61
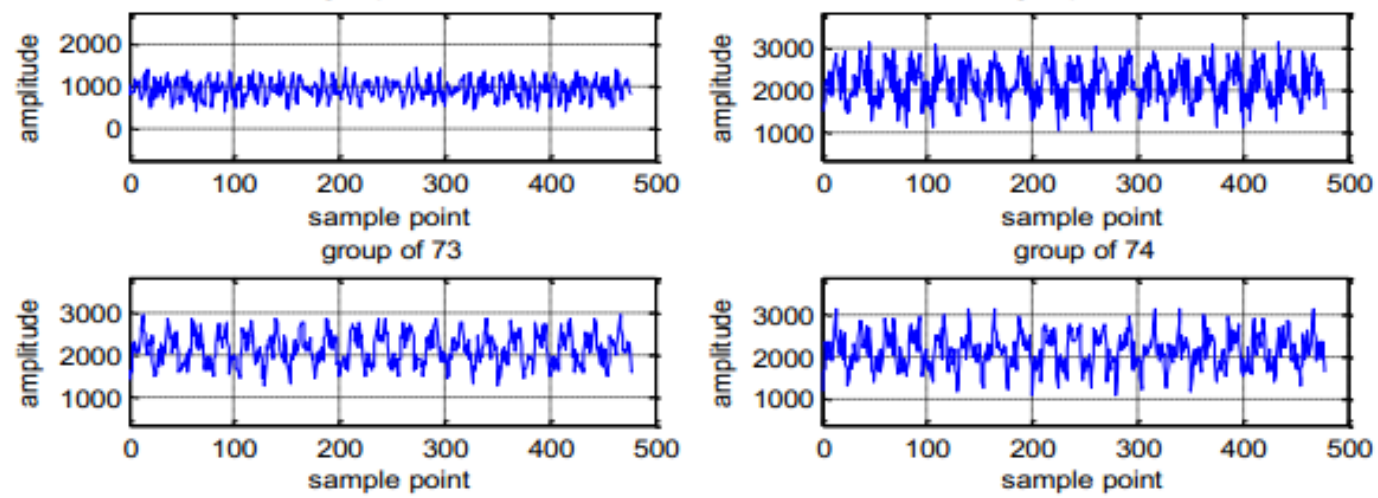

Figure 5 the spectrum change of the phase trajectory slope 


\section{Conclusion}

Based on the nonlinear dynamics theory, the theoretical analysis and simulation study about the sinusoidal signal, the active homing echo signal after filtering and the active homing echo signal after filtering and normalizing are made presented in this paper. Discussion of the three signals' phase trajectory change rule is given, also the phase trajectory slope change and the slope spectrum of the active homing echo signal after filtering and normalizing. Simulation results show that the phase trajectory of the active homing echo signal after treatment with the target echo signal is similar to the phase track motion rule of the sinusoidal signal; its phase trajectory slope is dense, continuous, regular short curve; and its spectrum changes similarly to the chaotic signal waveform. To the active homing echo signal after treatment which do not contain the target echo signal, its phase trajectory moves randomly in the phase plane; its phase trajectory slope is random distribution; its spectrum changes similarly to the white noise waveform, unorderly and irregularly. This proves that these nonlinear characteristics of the active homing echo signal have certain separability. The research result has a certain significance to the echo signal's characteristic identification.

\section{Reference}

[1] Huang Yongnian, An Introduction to Nonlinear Dynamics, Beijing University press, July 2010.

[2] Daniel Kaplan, Leon Glass. Understanding Nonlinear Dynamics, Springer Verlag, September 1997.

[3] Li Zhishun, The Torpedo Homing Signal and Information Processing, Northwestern polytechnical university press, May 2004

[4] Zhou Deshan, Li Zhishun, Zhu Bangyuan, Torpedo Homing Technology, National defense industry press, September 2009.

[5] Nie Chunyan, The Chaotic System and The Weak Signal Detection, Qinghua university press, March 2009.

[6] Li Hui, Chaotic Digital Communication, Beijing, Qinghua university press, 2006

[7] Zhang Defeng, MATLAB Simulation Communication Engineering, Beijing, Mechanical industry press, 2010. 